\title{
Dynamical analysis of almost periodic solution for a multispecies predator-prey model with mutual interference and time delays
}

\section{Qin Liu, Yuanfu Shao*, Si Zhou, Zhen Wang and Hairu Chen}

"Correspondence:

shaoyuanfu@163.com

School of Science, Guilin University

of Technology, Guilin, Guangxi

541004, P.R. China

\section{第 Springer}

\begin{abstract}
In this paper, we build a multispecies predator-prey model with mutual interference and time delays. By means of the comparison theorem, Ascoli theorem and Lebesgue dominated convergence theorem, we establish the sufficient conditions of permanence and investigate the existence of a unique almost periodic solution. By constructing a suitable Lyapunov function, we obtain that the positive almost periodic solution is globally attractive. Finally, we give numerical simulations to indicate the complex dynamical behaviors of this system.
\end{abstract}

Keywords: almost periodic solution; global attractivity; mutual interference; numerical simulation

\section{Introduction}

In population dynamics, the linkages between predator and prey are usually expressed by different functional response functions, which reflect different dynamical behaviors. Holling [1] carried out a large number of experiments on predator and prey and got some different functional response functions. For example, the mathematical expression of Holling $x_{i}(i=1,2)$ model is as follows [2]:

$$
\Phi(X)=\frac{\alpha X^{2}}{\beta^{2}+X^{2}} .
$$

Besides, in ecosystems, mutual interference between species is always present. The authors [3] proposed a mutual interference factor that tended to leave when the host or parasite met. A lot of articles studied the ecosystem with interference factors. Their obtained results showed that the effect of this factor should not be ignored [4-7]. For example, Wang et al. [6] concluded that mutual interference had great effect on the relative properties of predator-prey models.

In real life, time delay always exists. Food digestion time, resource regeneration time, mature time, pregnancy period and so on, these all can be expressed by time delay. Usually time delay plays a key role in many systems. For example, time delay can destroy the

(c) The Author(s) 2017. This article is distributed under the terms of the Creative Commons Attribution 4.0 International License (http://creativecommons.org/licenses/by/4.0/), which permits unrestricted use, distribution, and reproduction in any medium, provided you give appropriate credit to the original author(s) and the source, provide a link to the Creative Commons license, and indicate if changes were made. 
stability of the positive equilibrium. The obtained results showed that delayed differential equations exhibited more complex dynamical properties than ordinary differential equations [8-14]. Du et al. [10] gave the following model:

$$
\left\{\begin{array}{l}
\dot{x}=x(t)\left(r_{1}(t)-b_{1}(t) x(t-\tau(t))\right)-\frac{c_{1}(t) x^{2}(t)}{x^{2}(t)+k^{2}} y^{m}(t), \\
\dot{y}=y(t)\left(-r_{2}(t)-b_{2}(t) y(t)\right)+\frac{c_{2}(t) x^{2}(t)}{x^{2}(t)+k^{2}} y^{m}(t),
\end{array}\right.
$$

where all parameter meanings can be seen in [10]. The time delay of system (1.1) made the system very unstable and led to more complex dynamical behaviors. At the same time, the research methods were also very different from other systems.

From the point of view of the interaction between biology and environment, Darwin thought that biological variation, heredity and natural selection could lead to the adaptive change of organisms. We know that natural environment is not a constant, and organisms can change their habits to adapt to the new environment, which is called adaptive control. In recent years, adaptive control has been widely used in biological control systems, aerospace systems, satellite tracking systems, and so on $[15,16]$.

On the other hand, in Ref. [10], the authors assume that the coefficients $r_{1}(t), b_{1}(t), \tau(t)$, $c_{1}(t), r_{2}(t), b_{2}(t), c_{2}(t)$ of system (1.1) are continuous positive almost periodic functions. It is well known that the assumption of almost periodicity of the coefficients in (1.1) is a way of incorporating the time-dependent variability of the environment, especially when the factors of the environment exhibit periodical changes with not necessarily commensurate periods, such as weather, food, mating habits, harvest, etc. In view of these factors, it is necessary to study the relevant properties of ecosystems by using almost periodic coefficients. Recently, many scholars have studied the almost periodic solution and got some nice results, which showed that the almost periodic solution of a population dynamical system with mutual interference and time delay had wider application value [10, 17-19].

However, in the actual ecosystem, predator and prey always coexist, which is a common and widespread phenomenon. The dynamical property of a multispecies predator-prey system is much more complex than the system with only two or three species, and the analytical methods are very different [11, 20-22].

Based on the above discussion, we establish a multispecies predator-prey model with almost periodic coefficients, mutual interference and time delays. The corresponding mathematical model is as follows:

$$
\left\{\begin{array}{l}
x_{i}^{\prime}(t)=x_{i}(t)\left[r_{i}(t)-\sum_{k=1}^{n} b_{i k}(t) x_{k}\left(t-\tau_{k}(t)\right)-\sum_{k=1}^{m} \frac{c_{i k}(t) x_{i}(t)}{x_{i}^{2}(t)+f_{i k}(t)} y_{k}^{\alpha}(t)\right], \\
\quad i=1,2, \ldots, n, \\
y_{j}^{\prime}(t)=y_{j}(t)\left[-r_{j}(t)-\sum_{k=1}^{m} p_{j k}(t) y_{k}(t)+\sum_{k=1}^{n} \frac{q_{k j}(t) x_{k}^{2}(t)}{x_{k}^{2}(t)+f_{k j}(t)} y_{j}^{\alpha-1}(t)\right], \\
\quad j=1,2, \ldots, m,
\end{array}\right.
$$

with the initial conditions

$$
x_{i}(\chi)=\phi_{i}(\chi), \quad y_{j}(\chi)=\psi_{j}(\chi) ; \quad \phi_{i}(\chi), \psi_{j}(\chi) \in C\left([-\tau, 0], R_{+}\right), \chi \in[-\tau, 0],
$$

where $\tau=\max _{t \in R}\left\{\tau_{k}(t), k=1,2, \ldots, m\right\}, \tau_{k}(t)$ is a nonnegative and continuously differentiable almost periodic function on $R$ and $\min _{t \in R}\left\{1-\tau_{k}^{\prime}(t)\right\}>0 . r_{i}(t), b_{i k}(t), c_{i k}(t), f_{i k}(t), r_{j}(t)$, 
Table 1 Notations used to denote parameters

\begin{tabular}{ll}
\hline Parameters & Description \\
\hline$x_{j}(t)$ & The population of species of the ith prey at $t$. \\
$y_{j}(t)$ & The population of species of the $i$ th predator at $t$. \\
$r_{j}(t)$ & The population growth of prey without predators. \\
$r_{j}(t)$ & The decay rate of predator population without prey. \\
$\alpha$ & The mutual interference of predator and $0<\alpha<1$. \\
$b_{i k}(t)$ & The number of prey decreased due to inter-specific competition. \\
$p_{j k}(t)$ & The number of predator decreased due to inter-specific competition. \\
$c_{i k}(t)$ & The amount of prey eaten by predator. \\
$q_{k j}(t)$ & Conversion of energy from prey to predators. \\
\hline
\end{tabular}

$p_{j k}(t), q_{k j}(t), f_{k j}(t)$ are all continuous positive almost periodic functions on $R$ and the brief description about other parameters used in system (1.2) is presented in Table 1.

In this article, we aim to investigate the dynamical properties of almost periodic system (1.2), which can greatly enrich the biological background.

The structure of the article as follows. In Section 2, we introduce several important definitions and lemmas. We discuss the permanence of the system in Section 3. Next, we prove the global attractivity of system (1.2) in Section 4. In Section 5, we give conditions of the existence and uniqueness of almost periodic solutions for the system. We put numerical simulations in Section 6. In Section 7, we give a brief conclusion to this paper.

\section{Main descriptions}

In this part, we give some definitions and lemmas.

For continuous and bounded $f$ on $R$, we denote $f^{u}=\sup _{t \in R} f(t), f^{l}=\inf _{t \in R} f(t)$.

Definition 2.1 The positive solution $(x(t), y(t))^{T}=\left(x_{1}(t), x_{2}(t), \ldots, x_{n}(t), y_{1}(t), y_{2}(t), \ldots\right.$, $\left.y_{m}(t)\right)^{T}$ of system (1.2) is said to be globally attractive if, for any other positive solution $(\bar{x}(t), \bar{y}(t))^{T}=\left(\bar{x}_{1}(t), \bar{x}_{2}(t), \ldots, \bar{x}_{n}(t), \bar{y}_{1}(t), \bar{y}_{2}(t), \ldots, \bar{y}_{m}(t)\right)^{T}$ of (1.2), the following condition holds:

$$
\lim _{t \rightarrow+\infty}\left(\sum_{i=1}^{n}\left|x_{i}(t)-\bar{x}_{i}(t)\right|+\sum_{j=1}^{n}\left|y_{j}(t)-\bar{y}_{j}(t)\right|\right)=0 .
$$

Definition 2.2 ([23]) A function $f(t, x)$ is said to be almost periodic in $t$ uniformly with respect to $x \in X$ if $f(t, x)$ is continuous and, for $\forall \varepsilon>0$, it is possible to find a constant $I(\varepsilon)>0$ such that, for any interval of length $I(\varepsilon)$, there exists $\tau$ such that

$$
|f(t+\tau, x)-f(t, x)|<\varepsilon,
$$

where the number $\tau$ is called an $\varepsilon$-translation number of $f(t, x)$.

By the continuity of almost periodic functions, we obtain that the almost periodic coefficients satisfy $\min _{i=1,2, \ldots, n ; j=1,2, \ldots, m}\left\{r_{i}^{l}, r_{j}^{l}, b_{i k}^{l}, p_{j k}^{l}, c_{i k}^{l}, q_{k j}^{l}\right\}>0$ and $\max _{i=1,2, \ldots, n, j=1,2, \ldots, m}\left\{r_{i}^{u}, r_{j}^{u}, b_{i k}^{u}\right.$, $\left.p_{j k}^{u}, c_{i k}^{u}, q_{k j}^{u}\right\}<+\infty$. For, the characteristics and relevant definitions of almost periodic functions, the reader may refer to $[10,17,24]$. 
Definition 2.3 ([25]) An almost periodic function $f: R \rightarrow R$ is said to be asymptotic if there exist an almost periodic function $q(t)$ and a continuous function $r(t)$ such that

$$
f(t)=q(t)+r(t), \quad r(t) \rightarrow 0 \quad \text { as } t \rightarrow \infty .
$$

Lemma 2.1 ([26]) If the function $f(t)$ is nonnegative, integral and uniformly continuous on $[0,+\infty)$, then $\lim _{t \rightarrow \infty} f(t)=0$.

Lemma 2.2 The set $\left\{(x(t), y(t))^{T}=\left(x_{1}(t), x_{2}(t), \ldots, x_{n}(t), y_{1}(t), y_{2}(t), \ldots, y_{m}(t)\right)^{T} \in R^{n+m} \mid\right.$ $\left.x_{i}\left(t_{0}\right)>0, i=1,2, \ldots, n ; y_{j}\left(t_{0}\right)>0, j=1,2, \ldots, m, \exists t_{0} \in R\right\}$ is positive invariant with respect to system (1.2).

Proof For $x_{i}\left(t_{0}\right)>0, y_{j}\left(t_{0}\right)>0$, we have

$$
\begin{aligned}
& x_{i}(t)=x_{i}\left(t_{0}\right) \exp \left\{\int_{t_{0}}^{t}\left[r_{i}(s)-\sum_{k=1}^{n} b_{i k}(s) x_{k}\left(s-\tau_{k}(s)\right)-\sum_{k=1}^{m} \frac{c_{i k}(s) x_{i}(s)}{x_{i}^{2}(s)+f_{i k}(s)} y_{k}^{\alpha}(s)\right] d s\right\}>0, \\
& y_{j}(t)=y_{j}\left(t_{0}\right) \exp \left\{\int_{t_{0}}^{t}\left[-r_{j}(s)-\sum_{k=1}^{m} p_{j k}(s) y_{k}(s)+\sum_{k=1}^{n} \frac{q_{k j}(s) x_{k}^{2}(s)}{x_{k}^{2}(s)+f_{k j}(s)} y_{j}^{\alpha-1}(s)\right] d s\right\}>0 .
\end{aligned}
$$

Then Lemma 2.2 is obtained.

Lemma 2.3 ([27]) Suppose that the continuous operator A maps the closed and bounded convex set $Q \subset R^{n}$ onto itself, then the operator $A$ has at least one fixed point in the set $Q$.

Lemma 2.4 ([28]) If $x^{\prime} \geq(\leq) x\left(b-a x^{\alpha}\right)$, where $a>0, b>0$ and $\alpha$ is a positive constant, then

$$
\lim _{t \rightarrow \infty} \sup x(t) \leq\left(\frac{b}{a}\right)^{\frac{1}{\alpha}} \quad\left(\lim _{t \rightarrow \infty} \inf x(t) \geq\left(\frac{b}{a}\right)^{\frac{1}{\alpha}}\right) .
$$

Lemma 2.5 ([29]) If $x^{\prime} \geq(\leq) x^{m}(t)\left(b-a x^{1-m}(t)\right), x(0)>0, a>0, b>0$, then $\forall t \geq 0$, we have

$$
x(t) \geq(\leq)\left(\frac{b}{a}+\left(x^{1-m}(0)-\frac{b}{a}\right) e^{-a(1-m) t}\right)^{\frac{1}{1-m}} .
$$

\section{Permanence of system (1.2)}

Theorem 3.1 If the following condition holds:

$$
\left[H_{1}\right] \quad \hat{g}_{i}=r_{i}^{l}-\sum_{k=1, k \neq i}^{n} b_{i k}^{u}(t) M_{k}-\sum_{k=1}^{m} \frac{c_{i k}^{u} M_{i} N_{k}}{f_{i k}^{l}}>0,
$$

then system (1.2) is permanent, that is, there exists $T>0$, for $t>T>0$, the solution $(x(t), y(t))^{T}$ of $(1.2)$ satisfies $m_{i} \leq x_{i}(t) \leq M_{i}, n_{j} \leq y_{j}(t) \leq N_{j}$, where

$$
\begin{aligned}
& m_{i}=\frac{\hat{g}_{i}}{b_{i i}^{u}} \exp \left(\left(\hat{g}_{i}-b_{i i}^{u} M_{i}\right) \tau\right), \quad M_{i}=\frac{r_{i}^{u}}{b_{i i}^{l} e^{-r_{i}^{u} \tau}}, \\
& n_{j}=\left(\frac{\sum_{k=1}^{n} q_{k j}^{l} m_{k}^{2}}{2\left(\sum_{k=1}^{n}\left(M_{k}^{2}+f_{k j}^{u}\right)\right)\left(r_{j}^{u}+\sum_{k=1}^{m} p_{j k}^{u} N_{k}\right)}\right)^{\frac{1}{1-\alpha}}, \quad N_{j}=\left(\frac{3 \sum_{k=1}^{n} q_{k j}^{u}(t)}{2 r_{j}^{l}}\right)^{\frac{1}{1-\alpha}}
\end{aligned}
$$

for $i=1,2, \ldots, n ; j=1,2, \ldots, m$. In this article, the values of $i, j$ are no longer repeated. 
Proof By the first equation of (1.2), we get

$$
x_{i}^{\prime}(t) \leq x_{i}(t) r_{i}(t)
$$

Integrating (3.1), we have $x_{i}(t) \leq x_{i}(t-\tau) \exp \left(r_{i}^{u} \tau\right), t>\tau$, that is,

$$
x_{i}(t-\tau) \geq x_{i}(t) \exp \left(-r_{i}^{u} \tau\right), \quad t>\tau \text {. }
$$

Combining (3.2) and the first equation of (1.2), we have

$$
x_{i}^{\prime}(t) \leq x_{i}(t)\left[r_{i}^{u}-b_{i i}^{l} x_{i}(t) \exp \left(-r_{i}^{u} \tau\right)\right], \quad t>\tau .
$$

By applying Lemma 2.4 to (3.3), we obtain

$$
\lim _{t \rightarrow+\infty} \sup x_{i}(t) \leq \frac{r_{i}^{u}}{b_{i i}^{l} e^{-r_{i}^{u} \tau}} \equiv M_{i}
$$

By (3.4), there exists $T_{1}>\tau$, when $t \geq T_{1}$ and $T_{1} \rightarrow \infty$, then

$$
x_{i}(t) \leq M_{i}
$$

By (3.5), there also exists $T_{2}=T_{1}+\tau$, when $t \geq T_{2}$, then

$$
x_{i}(t-\tau) \leq M_{i}
$$

Combining (3.6) and the second equation of (1.2), we have

$$
\begin{aligned}
y_{j}^{\prime}(t) & \leq y_{j}(t)\left[\sum_{k=1}^{n} q_{k j}^{u}(t) y_{j}^{\alpha-1}(t)-r_{j}^{l}(t)\right] \\
& \leq y_{j}^{\alpha}(t)\left[\sum_{k=1}^{n} q_{k j}^{u}(t)-r_{j}^{l}(t) y^{1-\alpha}(t)\right], \quad t \geq T_{2} .
\end{aligned}
$$

Using Lemma 2.5 to (3.7), then

$$
y_{j}(t) \leq\left[\frac{\sum_{k=1}^{n} q_{k j}^{u}(t)}{r_{j}^{l}(t)}+\left(y^{1-\alpha}(0)-\frac{\sum_{k=1}^{n} q_{k j}^{u}(t)}{r_{j}^{l}(t)}\right) e^{-r_{j}^{l}(t)(1-\alpha) t}\right]^{\frac{1}{1-\alpha}}, \quad \forall t \geq 0 .
$$

Therefore, there exists $T_{3}>0$ such that

$$
y_{j}(t) \leq\left(\frac{3 \sum_{k=1}^{n} q_{k j}^{u}(t)}{2 r_{j}^{l}}\right)^{\frac{1}{1-\alpha}} \equiv N_{j}, \quad t>T_{3} .
$$

Combining (3.5), (3.6), (3.9) and the first equation of (1.2), we get

$$
x_{i}^{\prime}(t) \geq x_{i}(t)\left[r_{i}^{l}-\sum_{k=1, k \neq i}^{n} b_{i k}^{u}(t) M_{k}-b_{i i}^{u} x_{i}\left(t-\tau_{i}(t)\right)-\sum_{k=1}^{m} \frac{c_{i k}^{u} M_{i} N_{k}^{\alpha}}{f_{i k}^{l}}\right] .
$$


Suppose $x_{i}(\tilde{t})$ is any local minimal value of $x_{i}(t)$, then we have

$$
0=x_{i}^{\prime}(\tilde{t}) \geq x_{i}(\tilde{t})\left[r_{i}^{l}-\sum_{k=1, k \neq i}^{n} b_{i k}^{u}(t) M_{k}-b_{i i}^{u} x_{i}\left(\tilde{t}-\tau_{i}(\tilde{t})\right)-\sum_{k=1}^{m} \frac{c_{i k}^{u} M_{i} N_{k}^{\alpha}}{f_{i k}^{l}}\right] .
$$

Let

$$
\hat{g}_{i}=r_{i}^{l}-\sum_{k=1, k \neq i}^{n} b_{i k}^{u}(t) M_{k}-\sum_{k=1}^{m} \frac{c_{i k}^{u} M_{i} N_{k}^{\alpha}}{f_{i k}^{l}} .
$$

From (3.11) and (3.12), we have

$$
x_{i}\left(\tilde{t}-\tau_{i}(\tilde{t})\right) \geq \frac{\hat{g}_{i}}{b_{i i}^{u}} .
$$

Integrating (3.10) on $\left[\tilde{t}-\tau_{i}(\tilde{t}), \tilde{t}\right]$ and noticing that $\hat{g}_{i}-b_{i i}^{u} x_{i}\left(\tilde{t}-\tau_{i}(\tilde{t})\right) \leq 0$, we obtain

$$
\ln \left(\frac{x_{i}(\tilde{t})}{x_{i}\left(\tilde{t}-\tau_{i}(\tilde{t})\right)}\right) \geq \int_{\tilde{t}-\tau_{i}(\tilde{t})}^{\tilde{t}}\left(\hat{g}_{i}-b_{i i}^{u} x_{i}\left(\tilde{t}-\tau_{i}(\tilde{t})\right)\right) d t \geq\left(\hat{g}_{i}-b_{i i}^{u} M_{i}\right) \tau .
$$

From (3.13) and (3.14), then

$$
x_{i}(\tilde{t}) \geq \frac{\hat{g}_{i}}{b_{i i}^{u}} \exp \left(\left(\hat{g}_{i}-b_{i i}^{u} M_{i}\right) \tau\right) .
$$

Hence, for $T_{4}>0$ and $t>T_{4}$, we have

$$
x_{i}(t) \geq x_{i}(\tilde{t}) \geq \frac{\hat{g}_{i}}{b_{i i}^{u}} \exp \left(\left(\hat{g}_{i}-b_{i i}^{u} M_{i}\right) \tau\right) \equiv m_{i} .
$$

Combining (3.9), (3.16) and the second equation of (1.2), when $T_{5} \geq \max \left\{T_{3}, T_{4}\right\}>0$, for $t>T_{5}$, we get

$$
\begin{aligned}
y_{j}^{\prime}(t) & \geq y_{j}(t)\left[-r_{j}^{u}-\sum_{k=1}^{m} p_{j k}^{u} N_{k}+\sum_{k=1}^{n} \frac{q_{k j}^{l} m_{k}^{2}}{M_{k}^{2}+f_{k j}^{u}} y_{j}^{\alpha-1}(t)\right] \\
& =y_{j}^{\alpha}(t)\left[\sum_{k=1}^{n} \frac{q_{k j}^{l} m_{k}^{2}}{M_{k}^{2}+f_{k j}^{u}}-\left(r_{j}^{u}+\sum_{k=1}^{m} p_{j k}^{u} N_{k}\right) y_{j}^{1-\alpha}(t)\right] .
\end{aligned}
$$

It follows from Lemma 2.5 that there exists $T_{6}>0$ such that

$$
\begin{aligned}
y_{j}(t) & \geq\left(\frac{\sum_{k=1}^{n} \frac{q_{k j}^{l} m_{k}^{2}}{M_{k}^{2}+f_{k j}^{u}}}{r_{j}^{u}+\sum_{k=1}^{m} p_{j k}^{u} N_{k}}+\left(y_{j}^{1-\alpha}(0)-\frac{\sum_{k=1}^{n} \frac{q_{k j}^{l} m_{k}^{2}}{M_{k}^{2}+f_{j j}^{u}}}{r_{j}^{u}+\sum_{k=1}^{m} p_{j k}^{u} N_{k}}\right) e^{-\left(r_{j}^{u}+\sum_{k=1}^{m} p_{j k}^{u} N_{k}\right)(1-\alpha) t}\right)^{\frac{1}{1-\alpha}} \\
& \geq\left(\frac{\sum_{k=1}^{n} q_{k j}^{l} m_{k}^{2}}{2\left(\sum_{k=1}^{n}\left(M_{k}^{2}+f_{k j}^{u}\right)\right)\left(r_{j}^{u}+\sum_{k=1}^{m} p_{j k}^{u} N_{k}\right)}\right)^{\frac{1}{1-\alpha}} \equiv n_{j} .
\end{aligned}
$$

Make $T \geq \max \left\{T_{2}, T_{5}, T_{6}\right\}>0$, for $t>T$, we get $m_{i} \leq x_{i}(t) \leq M_{i}, n_{j} \leq y_{j}(t) \leq N_{j}$.

Therefore system (1.2) is permanent. 
Next, we prove that system (1.2) has at least one bounded positive solution for $t \geq 0$. Define $\Omega=\left\{(x(t), y(t))^{T}=\left(x_{1}(t), x_{2}(t), \ldots, x_{n}(t), y_{1}(t), y_{2}(t), \ldots, y_{m}(t)\right)^{T} \in R^{n+m} \mid(x(t), y(t))^{T}\right.$ is the solution of system (1.2), satisfying $\left.m_{i} \leq x_{i}(t) \leq M_{i}, n_{j} \leq y_{j}(t) \leq N_{j}, t \in R\right\}$.

Theorem 3.2 For system (1.2), the set $\Omega \neq \emptyset$.

Proof According to the characteristics of an almost periodic function, for a sequence of $\left\{t_{\gamma}\right\}, t_{\gamma} \rightarrow \infty$ as $\gamma \rightarrow \infty$, then $r_{i}\left(t+t_{\gamma}\right) \rightarrow r_{i}(t), r_{j}\left(t+t_{\gamma}\right) \rightarrow r_{j}(t), b_{i l}\left(t+t_{\gamma}\right) \rightarrow b_{i l}(t), p_{j k}(t+$ $\left.t_{\gamma}\right) \rightarrow p_{j k}(t), c_{i k}\left(t+t_{\gamma}\right) \rightarrow c_{i k}(t), q_{l j}\left(t+t_{\gamma}\right) \rightarrow q_{l j}(t), \tau_{i}\left(t+t_{\gamma}\right) \rightarrow \tau_{i}(t), f_{i j}\left(t+t_{\gamma}\right) \rightarrow f_{i j}(t)$ $(i, l=1,2, \ldots, n ; j, k=1,2, \ldots, m)$ uniformly on $R$ as $\gamma \rightarrow \infty$. By Lemma 2.3, system (1.2) has at least one solution $z(t)=(x(t), y(t))^{T}$ satisfying $m_{i} \leq x_{i}(t) \leq M_{i}, n_{j} \leq y_{j}(t) \leq N_{j}$ when $t>T$.

Obviously, the sequence $z\left(t+t_{\gamma}\right)$ is uniformly bounded and equi-continuous on any bounded subset of $R$. By the Ascoli theorem, we know there exists a subsequence $z\left(t+t_{\lambda}\right)$ which converges to a continuous function

$$
g(t)=\left(g_{1}(t), g_{2}(t)\right)^{T}=\left(g_{11}(t), g_{21}(t), \ldots, g_{n 1}(t), g_{12}(t), g_{22}(t), \ldots, g_{m 2}(t)\right)^{T}
$$

as $\lambda \rightarrow \infty$ uniformly on any bounded subset of $R$.

Make $T_{7} \in R$, suppose $T_{7}+t_{\lambda} \geq T$ for all $\lambda$. When $t \geq 0$, we obtain

$$
\begin{aligned}
x_{i}(t+ & \left.t_{\lambda}+T_{7}\right)-x_{i}\left(t_{\lambda}+T_{7}\right) \\
= & \int_{T_{7}}^{t+T_{7}} x_{i}\left(s+t_{\lambda}\right)\left(r_{i}\left(s+t_{\lambda}\right)-\sum_{k=1}^{n} b_{i k}\left(s+t_{\lambda}\right) x_{k}\left(\left(s+t_{\lambda}\right)-\tau_{k}\left(s+t_{\lambda}\right)\right)\right. \\
& \left.\quad-\sum_{k=1}^{m} \frac{c_{i k}\left(s+t_{\lambda}\right) x_{i}\left(s+t_{\lambda}\right)}{x_{i}^{2}\left(s+t_{\lambda}\right)+f_{i k}\left(s+t_{\lambda}\right)} y_{k}^{\alpha}\left(s+t_{\lambda}\right)\right) d s, \\
y_{j}(t+ & \left.t_{\lambda}+T_{7}\right)-y_{j}\left(t_{\lambda}+T_{7}\right) \\
= & \int_{T_{7}}^{t+T_{7}} y_{j}\left(t_{\lambda}+s\right)\left(-r_{j}\left(s+t_{\lambda}\right)-\sum_{k=1}^{m} p_{j k}\left(s+t_{\lambda}\right) y_{k}\left(s+t_{\lambda}\right)\right. \\
& \left.+\sum_{k=1}^{n} \frac{q_{k j}\left(s+t_{\lambda}\right) x_{k}^{2}\left(s+t_{\lambda}\right)}{x_{k}^{2}\left(s+t_{\lambda}\right)+f_{k j}\left(s+t_{\lambda}\right)} y_{j}^{\alpha-1}\left(s+t_{\lambda}\right)\right) d s .
\end{aligned}
$$

Letting $\lambda \rightarrow \infty$ in (3.17) and (3.18), for $\forall t \geq 0$, by the Lebesgue dominated convergence theorem, we get

$$
\begin{aligned}
& g_{i 1}\left(t+T_{7}\right)-g_{i 1}\left(T_{7}\right) \\
& \quad=\int_{T_{7}}^{t+T_{7}} g_{i 1}(s)\left(r_{i}(s)-\sum_{k=1}^{n} b_{i k}(s) g_{k 1}\left(s-\tau_{k}(s)\right)-\sum_{k=1}^{m} \frac{c_{i k}(s) g_{i 1}(s)}{g_{i 1}^{2}(s)+f_{i k}(s)} g_{k 2}^{\alpha}(s)\right) d s, \\
& g_{j 2}\left(t+T_{7}\right)-g_{j 2}\left(T_{7}\right) \\
& \quad=\int_{T_{7}}^{t+T_{7}} g_{j 2}(s)\left(-r_{j}(s)-\sum_{k=1}^{m} p_{j k}(s) g_{k 2}(s)+\sum_{k=1}^{n} \frac{q_{k j}(s) g_{k 1}^{2}(s)}{g_{k 1}^{2}(s)+f_{k j}(s)} g_{j 2}^{\alpha-1}(s)\right) d s .
\end{aligned}
$$

Since $T_{7} \in R$ is arbitrarily given, $g(t)$ is a solution of system (1.2) on $R$. 
It is easy to know $m_{i} \leq g_{i 1}(t) \leq M_{i}, n_{j} \leq g_{j 2}(t) \leq N_{j}$ for any $t \in R$. Thus, the set $\Omega \neq \emptyset$, that is, system (1.2) has at least one bounded positive solution.

\section{Global attractivity of system (1.2)}

Theorem 4.1 If the parameters of system (1.2) satisfy condition $\left[H_{1}\right]$ and the following conditions:

$$
\begin{aligned}
& {\left[H_{2}\right] \lim _{t \rightarrow+\infty} \inf A_{i}(t)>0,} \\
& {\left[H_{3}\right] \lim _{t \rightarrow+\infty} \inf B_{j}(t)>0,}
\end{aligned}
$$

where

$$
\begin{aligned}
& A_{i}(t)=-\sum_{k=1}^{m} c_{i k}(t) E_{i k}+\sum_{k=1}^{n} b_{i k}(t) \\
&-\sum_{k=1}^{n}\left[r_{k}(s)+\sum_{j=1}^{n} b_{k j}(s) M_{j}+\sum_{j=1}^{m} \frac{q_{k j}(s) M_{k} N_{j}^{\alpha}}{f_{k j}^{l}+m_{k}^{2}}\right] \int_{t}^{\varphi_{k}^{-1}(t)} b_{i k}(u) d u \\
&-\sum_{k=1}^{n} \sum_{j=1}^{m} c_{i k}(t) M_{k} E_{k j} \int_{t}^{\varphi_{k}^{-1}(t)} b_{i k}(u) d u \\
&-\sum_{k=1}^{n} \sum_{j=1}^{n} \frac{M_{k} b_{k j}\left(\varphi_{j}^{-1}(t)\right)}{1-\tau_{j}^{\prime}\left(\varphi_{j}^{-1}(l)\right)} \int_{\varphi_{j}^{-1}(t)}^{\varphi_{k}^{-1}\left(\varphi_{j}^{-1}(t)\right)} b_{i k}(u) d u-\sum_{k=1}^{m} q_{k j}(t) \frac{2 M_{i} N_{j}^{\alpha-1}}{f_{i k}(t)+m_{i}^{2}}, \\
& B_{j}(t)=-\sum_{k=1}^{m} c_{i k}(t) F_{i k}-\sum_{k=1}^{n} \sum_{j=1}^{m} q_{k j}(t) M_{k} E_{k j} \int_{t}^{\varphi_{k}^{-1}(t)} b_{i k}(u) d u+\sum_{k=1}^{m} p_{j k}(t), \\
& E_{i k}=\frac{f_{i k}^{u} N_{k}^{\alpha}+3 M_{i}^{2} N_{k}^{\alpha}}{\left(f_{i k}+m_{i}^{2}\right)^{2}}, \quad F_{i k}=\frac{f_{i k}^{u} M_{i}+M_{i}^{3}}{\left(f_{i k}+m_{i}^{2}\right)^{2}}
\end{aligned}
$$

and $\varphi_{i}^{-1}$ is the inverse function of $\varphi_{i}(t)=t-\tau_{i}(t)$, then the solution of system (1.2) is globally attractive.

Proof Let $(x(t), y(t))^{T},(\bar{x}(t), \bar{y}(t))^{T}$ be any two solutions of system (1.2). From Theorem 3.1, for $\forall t>T$, we get

$$
m_{i} \leq x_{i}(t), \bar{x}_{i}(t) \leq M_{i} ; \quad n_{j} \leq y_{j}(t), \bar{y}_{j}(t) \leq N_{j}
$$

Next, we set up several Lyapunov functions. Let

$$
V_{i 1}(t)=\left|\ln \bar{x}_{i}(t)-\ln x_{i}(t)\right|
$$

By calculating the upper right derivative of $V_{i 1}(t)$ along system (1.2), we have

$$
\begin{aligned}
D^{+} V_{i 1}(t) & =\operatorname{sign}\left(\bar{x}_{i}(t)-x_{i}(t)\right)\left(\frac{\bar{x}_{i}^{\prime}(t)}{\bar{x}_{i}(t)}-\frac{x_{i}^{\prime}(t)}{x_{i}(t)}\right) \\
& =\operatorname{sign}\left(\bar{x}_{i}(t)-x_{i}(t)\right)\left\{-\sum_{k=1}^{n} b_{i k}(t)\left[\bar{x}_{k}\left(t-\tau_{k}(t)\right)-x_{k}\left(t-\tau_{k}(t)\right)\right]\right.
\end{aligned}
$$




$$
\begin{aligned}
& \left.-\sum_{k=1}^{m}\left[\frac{c_{i k}(t) \bar{x}_{i}(t) \bar{y}_{k}^{\alpha}(t)}{\bar{x}_{i}^{2}(t)+f_{i k}(t)}-\frac{c_{i k}(t) x_{i}(t) y_{k}^{\alpha}(t)}{x_{i}^{2}(t)+f_{i k}(t)}\right]\right\} \\
= & \operatorname{sign}\left(\bar{x}_{i}(t)-x_{i}(t)\right)\left\{-\sum_{k=1}^{n} b_{i k}(t)\left[\bar{x}_{k}\left(t-\tau_{k}(t)\right)-x_{k}\left(t-\tau_{k}(t)\right)\right]\right. \\
& -\sum_{k=1}^{m} c_{i k}(t)\left[\frac{f_{i k}(t) \bar{y}_{k}^{\alpha}(t)+\bar{y}_{k}^{\alpha}(t) x_{i}^{2}(t)-x_{i}(t) y_{k}^{\alpha}(t)\left(x_{i}(t)+\bar{x}_{i}(t)\right)}{\left(\bar{x}_{i}^{2}(t)+f_{i k}(t)\right)\left(x_{i}^{2}(t)+f_{i k}(t)\right)}(t)-x_{i}(t)\right) \\
& \left.\left.+\frac{f_{i k}(t) x_{i}(t)+x_{i}^{3}(t)}{\left(\bar{x}_{i}^{2}(t)+f_{i k}(t)\right)\left(x_{i}^{2}(t)+f_{i k}(t)\right)}\left(\bar{y}_{k}^{\alpha}(t)-y_{k}^{\alpha}(t)\right)\right]\right\} \\
\leq & \sum_{k=1}^{m} c_{i k}(t) E_{i k}\left|\bar{x}_{i}(t)-x_{i}(t)\right|+\sum_{k=1}^{m} c_{i k}(t) F_{i k}\left|\bar{y}_{k}^{\alpha}(t)-y_{k}^{\alpha}(t)\right| \\
& -\sum_{k=1}^{n} b_{i k}\left|\bar{x}_{k}(t)-x_{k}(t)\right|+\sum_{k=1}^{n} b_{i k}\left|\int_{t-\tau_{k}(t)}^{t} \bar{x}_{k}^{\prime}(s)-x_{k}^{\prime}(s) d s\right|,
\end{aligned}
$$

where

$$
E_{i k}=\frac{f_{i k}^{u} N_{k}^{\alpha}+3 M_{i}^{2} N_{k}^{\alpha}}{\left(f_{i k}+m_{i}^{2}\right)^{2}}, \quad F_{i k}=\frac{f_{i k}^{u} M_{i}+M_{i}^{3}}{\left(f_{i k}+m_{i}^{2}\right)^{2}} .
$$

Substituting (1.2) into (4.3), we get

$$
\begin{aligned}
D^{+} V_{i 1}(t) & \\
\leq & \sum_{k=1}^{m} c_{i k}(t) E_{i k}\left|\bar{x}_{i}(t)-x_{i}(t)\right|+\sum_{k=1}^{m} c_{i k}(t) F_{i k}\left|\bar{y}_{k}^{\alpha}(t)-y_{k}^{\alpha}(t)\right|-\sum_{k=1}^{n} b_{i k}(t)\left|\bar{x}_{k}(t)-x_{k}(t)\right| \\
& +\sum_{k=1}^{n} b_{i k}(t) \mid \int_{t-\tau_{k}(t)}^{t}\left\{\bar{x}_{k}(s)\left[r_{k}(s)-\sum_{j=1}^{n} b_{k j}(s) \bar{x}_{j}\left(s-\tau_{j}(s)\right)-\sum_{j=1}^{m} \frac{c_{k j}(s) \bar{x}_{k}(s) \bar{y}_{j}^{\alpha}(s)}{f_{k j}(s)+\bar{x}_{k}^{2}(s)}\right]\right. \\
& \left.-x_{k}(s)\left[r_{k}(s)-\sum_{j=1}^{n} b_{k j}(s) x_{j}\left(s-\tau_{j}(s)\right)-\sum_{j=1}^{m} \frac{c_{k j}(s) x_{k}(s) y_{j}^{\alpha}(s)}{f_{k j}(s)+x_{k}^{2}(s)}\right]\right\} d s \mid \\
= & \sum_{k=1}^{m} c_{i k}(t) E_{i k}\left|\bar{x}_{i}(t)-x_{i}(t)\right|+\sum_{k=1}^{m} c_{i k}(t) F_{i k}\left|\bar{y}_{k}^{\alpha}(t)-y_{k}^{\alpha}(t)\right|-\sum_{k=1}^{n} b_{i k}(t) E_{i k}\left|\bar{x}_{k}(t)-x_{k}(t)\right| \\
& +\sum_{k=1}^{n} b_{i k}(t) \mid \int_{t-\tau_{k}(t)}^{t}\left\{\left[r_{k}(s)-\sum_{j=1}^{n} b_{k j}(s) \bar{x}_{j}\left(s-\tau_{j}(s)\right)-\sum_{j=1}^{m} \frac{c_{k j}(s) \bar{x}_{k}(s) \bar{y}_{j}^{\alpha}(s)}{f_{k j}(s)+\bar{x}_{k}^{2}(s)}\right]\right. \\
& \times\left(\bar{x}_{k}(s)-x_{k}(s)\right)-x_{k}(s) \sum_{j=1}^{n} b_{k j}(s)\left(\bar{x}_{j}\left(s-\tau_{j}(s)\right)-x_{j}\left(s-\tau_{j}(s)\right)\right) \\
& -x_{k}(s) \sum_{j=1}^{m} c_{k j}(s) \frac{f_{k j}(s) \bar{y}_{j}^{\alpha}(s)+\bar{y}_{j}^{\alpha}(s) x_{k}^{2}(s)-y_{j}^{\alpha}(s) x_{k}(s)\left(x_{k}(s)+\bar{x}_{k}(s)\right)}{\left(f_{k j}(s)+x_{k}^{2}(s)\right)\left(f_{k j}(s)+\bar{x}_{k}^{2}(s)\right)} \\
& \left.-x_{k}(s) \sum_{j=1}^{m} c_{k j}(s) \frac{f_{k j}(s) x_{k}(s)+x_{k}^{3}(s)}{\left(f_{k j}(s)+x_{k}^{2}(s)\right)\left(f_{k j}(s)+\bar{x}_{k}^{2}(s)\right)}\left(\bar{y}_{j}^{\alpha}(s)-y_{j}^{\alpha}(s)\right)\right\} d s \mid
\end{aligned}
$$




$$
\begin{aligned}
\leq & \sum_{k=1}^{m} c_{i k}(t) E_{i k}\left|\bar{x}_{i}(t)-x_{i}(t)\right|+\sum_{k=1}^{m} c_{i k}(t) F_{i k}\left|\bar{y}_{k}^{\alpha}(t)-y_{k}^{\alpha}(t)\right|-\sum_{k=1}^{n} b_{i k}(t)\left|\bar{x}_{k}(t)-x_{k}(t)\right| \\
& +\sum_{k=1}^{n} b_{i k}(t) \int_{t-\tau_{k}(t)}^{t}\left\{\left[r_{k}(s)+\sum_{j=1}^{n} b_{k j}(s) \bar{x}_{j}\left(s-\tau_{j}(s)\right)+\sum_{j=1}^{m} \frac{c_{k j}(s) \bar{x}_{k}(s) \bar{y}_{j}^{\alpha}(s)}{f_{k j}(s)+\bar{x}_{k}^{2}(s)}\right]\right. \\
& \times\left|\bar{x}_{k}(s)-x_{k}(s)\right|+x_{k}(s) \sum_{j=1}^{n} b_{k j}(s)\left|\left(\bar{x}_{j}\left(s-\tau_{j}(s)\right)-x_{j}\left(s-\tau_{j}(s)\right)\right)\right| \\
& \left.+x_{k}(s) \sum_{j=1}^{m} c_{k j}(s) E_{k j}\left|\bar{x}_{k}(s)-x_{k}(s)\right|+x_{k}(s) \sum_{j=1}^{m} c_{k j}(s) F_{k j}\left|\bar{y}_{j}^{\alpha}(s)-y_{j}^{\alpha}(s)\right|\right\} d s .
\end{aligned}
$$

Considering (4.1) and (4.4), for $t \geq T+\tau$, we get

$$
\begin{aligned}
& D^{+} V_{i 1}(t) \\
& \leq \sum_{k=1}^{m} c_{i k}(t) E_{i k}\left|\bar{x}_{i}(t)-x_{i}(t)\right|+\sum_{k=1}^{m} c_{i k}(t) F_{i k}\left|\bar{y}_{k}^{\alpha}(t)-y_{k}^{\alpha}(t)\right|-\sum_{k=1}^{n} b_{i k}(t)\left|\bar{x}_{k}(t)-x_{k}(t)\right| \\
& \quad+\sum_{k=1}^{n} b_{i k}(t) \int_{t-\tau_{k}(t)}^{t}\left\{\left[r_{k}(s)+\sum_{j=1}^{n} b_{k j}(s) M_{j}+\sum_{j=1}^{m} \frac{c_{k j}(s) M_{k} N_{j}^{\alpha}}{f_{k j}^{l}+m_{k}^{2}}\right]\left|\bar{x}_{k}(s)-x_{k}(s)\right|\right. \\
& \quad+M_{k} \sum_{j=1}^{n} b_{k j}(s)\left|\left(\bar{x}_{j}\left(s-\tau_{j}(s)\right)-x_{j}\left(s-\tau_{j}(s)\right)\right)\right| \\
& \left.\quad+M_{k} \sum_{j=1}^{n} c_{k j}(s) E_{k j}\left|\bar{x}_{k}(s)-x_{k}(s)\right|+M_{k} \sum_{j=1}^{m} c_{k j}(s) F_{k j}\left|\bar{y}_{j}^{\alpha}(s)-y_{j}^{\alpha}(s)\right|\right\} d s \\
& \equiv \sum_{k=1}^{m} c_{i k}(t) E_{i k}\left|\bar{x}_{i}(t)-x_{i}(t)\right|+\sum_{k=1}^{m} c_{i k}(t) F_{i k}\left|\bar{y}_{k}^{\alpha}(t)-y_{k}^{\alpha}(t)\right|-\sum_{k=1}^{n} b_{i k}(t)\left|\bar{x}_{k}(t)-x_{k}(t)\right| \\
& \quad+\sum_{k=1}^{n} b_{i k}(t) \int_{t-\tau_{k}(t)}^{t} G_{k}(s) d s .
\end{aligned}
$$

Define

$$
V_{i 2}(t)=\sum_{k=1}^{n} \int_{t}^{\varphi_{k}^{-1}(t)} \int_{\tau_{k}(u)}^{t} b_{i k}(u) G_{k}(s) d s d u .
$$

Combining (4.5) and (4.6), for $t \geq T+\tau$, we get

$$
\begin{aligned}
D^{+} V_{i 1}(t)+V_{i 2}^{\prime}(t) \leq & \sum_{k=1}^{m} c_{i k}(t) E_{i k}\left|\bar{x}_{i}(t)-x_{i}(t)\right| \\
& +\sum_{k=1}^{m} c_{i k}(t) F_{i k}\left|\bar{y}_{k}^{\alpha}(t)-y_{k}^{\alpha}(t)\right|-\sum_{k=1}^{n} b_{i k}(t)\left|\bar{x}_{k}(t)-x_{k}(t)\right| \\
& +\sum_{k=1}^{n} \int_{t}^{\varphi_{k}^{-1}(t)} b_{i k}(u) d u G_{k}(t) .
\end{aligned}
$$


Next, we define

$$
V_{i}(t)=V_{i 1}(t)+V_{i 2}(t)+V_{i 3}(t)
$$

where

$$
V_{i 3}(t)=\sum_{k=1}^{n} \sum_{j=1}^{n} M_{k} \int_{t-\tau_{j}(t)}^{t} \int_{\varphi_{j}^{-1}(l)}^{\varphi_{k}^{-1}\left(\varphi_{j}^{-1}(l)\right)} \frac{b_{i k}(u) b_{k j}\left(\varphi_{j}^{-1}(l)\right)}{1-\tau_{j}^{\prime}\left(\varphi_{j}^{-1}(l)\right)}\left|\bar{x}_{j}(l)-x_{j}(l)\right| d u d l .
$$

Considering (4.7)-(4.9), for $t \geq T+\tau$, we get

$$
\begin{aligned}
D^{+} V_{i}(t) & \\
\leq & \sum_{k=1}^{m} c_{i k}(t) E_{i k}\left|\bar{x}_{i}(t)-x_{i}(t)\right|+\sum_{k=1}^{m} c_{i k}(t) F_{i k}\left|\bar{y}_{k}^{\alpha}(t)-y_{k}^{\alpha}(t)\right|-\sum_{k=1}^{n} b_{i k}(t)\left|\bar{x}_{k}(t)-x_{k}(t)\right| \\
& +\sum_{k=1}^{n}\left[r_{k}(s)+\sum_{j=1}^{n} b_{k j}(s) M_{j}+\sum_{j=1}^{m} \frac{c_{k j}(s) M_{k} N_{j}^{\alpha}}{f_{k j}^{l}+m_{k}^{2}}\right] \int_{t}^{\varphi_{k}^{-1}(t)} b_{i k}(u) d u\left|\bar{x}_{k}(t)-x_{k}(t)\right| \\
& +\sum_{k=1}^{n} \sum_{j=1}^{m} c_{i k}(t) M_{k} E_{k j} \int_{t}^{\varphi_{k}^{-1}(t)} b_{i k}(u) d u\left|\bar{x}_{k}(t)-x_{k}(t)\right| \\
& +\sum_{k=1}^{n} \sum_{j=1}^{m} c_{k j}(t) M_{k} F_{k j} \int_{t}^{\varphi_{k}^{-1}(t)} b_{i k}(u) d u\left|\bar{y}_{j}^{\alpha}(t)-y_{j}^{\alpha}(t)\right| \\
& +\sum_{k=1}^{n} \sum_{j=1}^{n} \frac{M_{k} b_{k j}\left(\varphi_{j}^{-1}(t)\right)}{1-\tau_{j}^{\prime}\left(\varphi_{j}^{-1}(l)\right)} \int_{\varphi_{j}^{-1}(t)}^{\varphi_{k}^{-1}\left(\varphi_{j}^{-1}(t)\right)} b_{i k}(u) d u\left|\bar{x}_{j}(t)-x_{j}(t)\right| .
\end{aligned}
$$

Define

$$
V_{j}(t)=\left|\ln y_{j}(t)-\ln \bar{y}_{j}(t)\right|
$$

Calculating its Dini derivative along system (1.2), we get

$$
\begin{aligned}
D^{+} V_{j}(t)= & \operatorname{sign}\left(y_{j}(t)-\bar{y}_{j}(t)\right)\left(\frac{y_{j}^{\prime}(t)}{y_{j}(t)}-\frac{\bar{y}_{j}^{\prime}(t)}{\bar{y}_{j}(t)}\right) \\
= & \operatorname{sign}\left(y_{j}(t)-\bar{y}_{j}(t)\right)\left[-\sum_{k=1}^{m} p_{j k}(t)\left(y_{j}(t)-\bar{y}_{j}(t)\right)\right. \\
& \left.+\sum_{k=1}^{m} q_{k j}(t)\left(\frac{x_{k}^{2}(t) y_{j}^{\alpha-1}(t)}{f_{i k}(t)+x_{k}^{2}(t)}-\frac{\bar{x}_{k}^{2}(t) \bar{y}_{j}^{\alpha-1}(t)}{f_{i k}(t)+\bar{x}_{k}^{2}(t)}\right)\right] \\
= & -\sum_{k=1}^{m} p_{j k}(t)\left|y_{j}(t)-\bar{y}_{j}(t)\right| \\
& +\sum_{k=1}^{m} q_{k j}(t) \operatorname{sign}\left(y_{j}(t)-\bar{y}_{j}(t)\right)\left(\frac{x_{k}^{2}(t) y_{j}^{\alpha-1}(t)}{f_{i k}(t)+x_{k}^{2}(t)}-\frac{\bar{x}_{k}^{2}(t) \bar{y}_{j}^{\alpha-1}(t)}{f_{i k}(t)+\bar{x}_{k}^{2}(t)}\right) \\
= & -\sum_{k=1}^{m} p_{j k}(t)\left|y_{j}(t)-\bar{y}_{j}(t)\right|+\sum_{k=1}^{m} q_{k j}(t) \operatorname{sign}\left(y_{j}(t)-\bar{y}_{j}(t)\right)
\end{aligned}
$$




$$
\begin{aligned}
& \times\left(\frac{x_{k}^{2}(t) y_{j}^{\alpha-1}(t)}{f_{i k}(t)+x_{k}^{2}(t)}-\frac{x_{k}^{2}(t) \bar{y}_{j}^{\alpha-1}(t)}{f_{i k}(t)+x_{k}^{2}(t)}+\frac{x_{k}^{2}(t) \bar{y}_{j}^{\alpha-1}(t)}{f_{i k}(t)+x_{k}^{2}(t)}-\frac{\bar{x}_{k}^{2}(t) \bar{y}_{j}^{\alpha-1}(t)}{f_{i k}(t)+\bar{x}_{k}^{2}(t)}\right) \\
= & -\sum_{k=1}^{m} p_{j k}(t)\left|y_{j}(t)-\bar{y}_{j}(t)\right|+\sum_{k=1}^{m} q_{k j}(t) \operatorname{sign}\left(y_{j}(t)-\bar{y}_{j}(t)\right) \\
& \times\left(\frac{x_{k}^{2}(t)}{f_{i k}(t)+x_{k}^{2}(t)}\left(y_{j}^{\alpha-1}(t)-\bar{y}_{j}^{\alpha-1}(t)\right)\right. \\
& \left.+\frac{f_{i k}(t)\left(x_{k}(t)+\bar{x}_{k}(t)\right) \bar{y}_{j}^{\alpha-1}(t)}{\left(f_{i k}(t)+x_{k}^{2}(t)\right)\left(f_{i k}(t)+\bar{x}_{k}^{2}(t)\right)}\left(x_{k}(t)-\bar{x}_{k}(t)\right)\right) \\
\leq & -\sum_{k=1}^{m} p_{j k}(t)\left|y_{j}(t)-\bar{y}_{j}(t)\right|+\sum_{k=1}^{m} q_{k j}(t) \frac{\left(x_{k}(t)+\bar{x}_{k}(t)\right) \bar{y}_{j}^{\alpha-1}(t)}{f_{i k}(t)+x_{k}^{2}(t)}\left|x_{k}(t)-\bar{x}_{k}(t)\right| \\
\leq & -\sum_{k=1}^{m} p_{j k}(t)\left|y_{j}(t)-\bar{y}_{j}(t)\right|+\sum_{k=1}^{m} q_{k j}(t) \frac{2 M_{i} N_{j}^{\alpha-1}}{f_{i k}(t)+m_{i}^{2}}\left|x_{k}(t)-\bar{x}_{k}(t)\right| .
\end{aligned}
$$

Define the Lyapunov functional $V(t)$ as follows:

$$
V(t)=\sum_{i=1}^{n} V_{i}(t)+\sum_{j=1}^{m} V_{j}(t)
$$

Considering (4.10), (4.11) and (4.12), for $t \geq T+\tau$, we have

$$
D^{+} V(t) \leq-\sum_{i=1}^{n} A_{i}(t)\left|\bar{x}_{i}(t)-x_{i}(t)\right|-\sum_{j=1}^{m} B_{j}(t)\left|\bar{y}_{j}(t)-y_{j}(t)\right|
$$

where $A_{i}(t), B_{j}(t)$ are given in Theorem 4.1.

From conditions $\left[H_{2}\right]$ and $\left[H_{3}\right]$, there exist $\alpha_{i}, \beta_{j}>0$ and $T_{0} \geq T+\tau$ such that

$$
0<\alpha_{i} \leq A_{i}(t), \quad 0<\beta_{j} \leq B_{j}(t) \quad \text { for } t \geq T_{0} .
$$

Let $\alpha_{0}=\min \left\{\alpha_{1}, \alpha_{2}, \ldots, \alpha_{n} ; \beta_{1}, \beta_{2}, \ldots, \beta_{m}\right\}$, combining (4.14) and (4.15), then

$$
D^{+} V(t) \leq-\alpha_{0}\left(\sum_{i=1}^{n}\left|\bar{x}_{i}(t)-x_{i}(t)\right|+\sum_{j=1}^{m}\left|\bar{y}_{j}(t)-y_{j}(t)\right|\right) .
$$

Integrating (4.16) on $\left[T_{0}, t\right]$, we get

$$
V(t)+\alpha_{0} \int_{T_{0}}^{t}\left(\sum_{i=1}^{n}\left|\bar{x}_{i}(u)-x_{i}(u)\right|+\sum_{j=1}^{m}\left|\bar{y}_{j}(u)-y_{j}(u)\right|\right) d u \leq V\left(T_{0}\right), \quad t \geq T_{0} .
$$

So, $\int_{T_{0}}^{+\infty}\left(\sum_{i=1}^{n}\left|\bar{x}_{i}(u)-x_{i}(u)\right|+\sum_{j=1}^{m}\left|\bar{y}_{j}(u)-y_{j}(u)\right|\right) d u<+\infty$ and $V(t)$ is bounded on the interval $\left[T_{0},+\infty\right)$. Combining Theorem 3.1 and (1.2), we get $\bar{x}_{i}(t)-x_{i}(t), \bar{y}_{j}(t)-y_{j}(t)$ and $\left(\bar{x}_{i}(t)-x_{i}(t)\right)^{\prime},\left(\bar{y}_{j}(t)-y_{j}(t)\right)^{\prime}$ are bounded on the interval $\left[T_{0},+\infty\right)$. Then $\sum_{i=1}^{n}\left|\bar{x}_{i}(u)-x_{i}(u)\right|+$ $\sum_{j=1}^{m}\left|\bar{y}_{j}(u)-y_{j}(u)\right|$ is uniformly continuous. 
Using Lemma 2.1, we get

$$
\begin{aligned}
& \lim _{t \rightarrow+\infty}\left(\sum_{i=1}^{n}\left|\bar{x}_{i}(t)-x_{i}(t)\right|+\sum_{j=1}^{m}\left|\bar{y}_{j}(t)-y_{j}(t)\right|\right)=0 \quad \text { and } \\
& \lim _{t \rightarrow+\infty}\left|\bar{x}_{i}(t)-x_{i}(t)\right|=0, \quad \lim _{t \rightarrow+\infty}\left|\bar{y}_{j}(t)-y_{j}(t)\right|=0 .
\end{aligned}
$$

Therefore, system (1.2) is globally attractive.

\section{Existence of almost periodic solution}

Theorem 5.1 Suppose $\left[H_{1}\right],\left[H_{2}\right]$ and $\left[H_{3}\right]$ hold, then system (1.2) has a unique almost periodic solution.

Proof From Theorem 3.2, we know $(x(t), y(t))^{T}, t \in R$ is a bounded positive solution. Then there exists a sequence $\left\{t_{\lambda}^{\prime}\right\}, t_{\lambda}^{\prime} \rightarrow \infty$ as $\lambda \rightarrow+\infty$ such that $\left(x\left(t+t_{\lambda}^{\prime}\right), y\left(t+t_{\lambda}^{\prime}\right)\right)^{T}$ is a solution of the following system (5.1):

$$
\left\{\begin{array}{l}
x_{i}^{\prime}(t)=x_{i}(t)\left[r_{i}\left(t+t_{\lambda}^{\prime}\right)-\sum_{k=1}^{n} b_{i k}\left(t+t_{\lambda}^{\prime}\right) x_{k}\left(t-\tau_{k}(t)\right)-\sum_{k=1}^{m} \frac{c_{i k}\left(t+t_{\lambda}^{\prime}\right) x_{i}(t)}{x_{i}^{2}(t)+f_{i k}\left(t+t_{\lambda}^{\prime}\right)} y_{k}^{\alpha}(t)\right] \\
y_{j}^{\prime}(t)=y_{j}(t)\left[-r_{j}\left(t+t_{\lambda}^{\prime}\right)-\sum_{k=1}^{m} p_{j k}\left(t+t_{\lambda}^{\prime}\right) y_{k}(t)+\sum_{k=1}^{n} \frac{q_{k j}\left(t+t_{\lambda}^{\prime}\right) x_{k}^{2}(t)}{x_{k}^{2}(t)+f_{k j}\left(t+t_{\lambda}^{\prime}\right)} y_{j}^{\alpha-1}(t)\right] .
\end{array}\right.
$$

From the above and Theorem 3.1, we know $\left(x\left(t+t_{\lambda}^{\prime}\right), y\left(t+t_{\lambda}^{\prime}\right)\right)^{T}$ and $\left(x^{\prime}\left(t+t_{\lambda}^{\prime}\right), y^{\prime}\left(t+t_{\lambda}^{\prime}\right)\right)^{T}$ are uniformly bounded. Clearly, the sequence $\left(x\left(t+t_{\lambda}^{\prime}\right), y\left(t+t_{\lambda}^{\prime}\right)\right)^{T}$ is equi-continuous. By the Ascoli theorem, there exists a uniformly convergent subsequence $\left\{\left(x\left(t+t_{\lambda}\right), y\left(t+t_{\lambda}\right)\right)^{T}\right\} \subseteq$ $\left\{\left(x\left(t+t_{\lambda}^{\prime}\right), y\left(t+t_{\lambda}^{\prime}\right)\right)^{T}\right\}$ such that, for any $\forall \varepsilon>0$, there exists $\lambda_{0}(\varepsilon)>0$ with the property that if $\lambda, \varpi>\lambda_{0}(\varepsilon)$, then

$$
\left|x_{i}\left(t+t_{\varpi}\right)-x_{i}\left(t+t_{\lambda}\right)\right|<\varepsilon, \quad\left|y_{j}\left(t+t_{\varpi}\right)-y_{j}\left(t+t_{\lambda}\right)\right|<\varepsilon,
$$

which indicates that $\left(x\left(t+t_{\lambda}\right), y\left(t+t_{\lambda}\right)\right)^{T}$ is an almost periodic and asymptotic function. Then there exist functions $g_{i 1}(t), g_{j 2}(t), h_{i 1}(t), h_{j 2}(t)$ such that

$$
x_{i}(t)=g_{i 1}(t)+h_{i 1}(t), \quad y_{j}(t)=g_{j 2}(t)+h_{j 2}(t),
$$

where

$$
\begin{array}{ll}
\lim _{\lambda \rightarrow+\infty} g_{i 1}\left(t+t_{\lambda}\right)=g_{i 1}(t), & \lim _{\lambda \rightarrow+\infty} g_{j 2}\left(t+t_{\lambda}\right)=g_{j 2}(t), \\
\lim _{\lambda \rightarrow+\infty} h_{i 1}\left(t+t_{\lambda}\right)=0, & \lim _{\lambda \rightarrow+\infty} h_{j 2}\left(t+t_{\lambda}\right)=0,
\end{array}
$$

$g_{i 1}(t), g_{j 2}(t)$ are almost periodic functions. It shows that

$$
\lim _{\lambda \rightarrow+\infty} x_{i}\left(t+t_{\lambda}\right)=g_{i 1}(t), \quad \lim _{\lambda \rightarrow+\infty} y_{j}\left(t+t_{\lambda}\right)=g_{j 2}(t) .
$$

Besides, we still have

$$
\lim _{\lambda \rightarrow+\infty} x_{i}^{\prime}\left(t+t_{\lambda}\right)=\lim _{\lambda \rightarrow+\infty} \lim _{\bar{h} \rightarrow 0} \frac{x_{i}\left(t+t_{\lambda}+\bar{h}\right)-x_{i}\left(t+t_{\lambda}\right)}{\bar{h}}=\lim _{\bar{h} \rightarrow 0} \frac{g_{i 1}(t+\bar{h})-g_{i 1}(t)}{\bar{h}},
$$




$$
\lim _{\lambda \rightarrow+\infty} y_{j}^{\prime}\left(t+t_{\lambda}\right)=\lim _{\lambda \rightarrow+\infty} \lim _{\bar{h} \rightarrow 0} \frac{y_{j}\left(t+t_{\lambda}+\bar{h}\right)-y_{j}\left(t+t_{\lambda}\right)}{\bar{h}}=\lim _{\bar{h} \rightarrow 0} \frac{g_{j 2}(t+\bar{h})-g_{j 2}(t)}{\bar{h}} .
$$

Therefore, the derivatives $g_{i 1}^{\prime}(t), g_{j 2}^{\prime}(t)$ exist.

Next, we prove $g(t)=\left(g_{1}(t), g_{2}(t)\right)^{T}$ is an almost periodic solution of system (1.2).

By the characteristics of almost periodic solution, there exists a sequence $\left\{t_{\gamma}\right\}, t_{\gamma} \rightarrow$ $\infty$ as $\gamma \rightarrow+\infty$ such that $r_{i}\left(t+t_{\gamma}\right) \rightarrow r_{i}(t), r_{j}\left(t+t_{\gamma}\right) \rightarrow r_{j}(t), b_{i l}\left(t+t_{\gamma}\right) \rightarrow b_{i l}(t), p_{j k}(t+$ $\left.t_{\gamma}\right) \rightarrow p_{j k}(t), c_{i k}\left(t+t_{\gamma}\right) \rightarrow c_{i k}(t), q_{l j}\left(t+t_{\gamma}\right) \rightarrow q_{l j}(t), \tau_{i}\left(t+t_{\gamma}\right) \rightarrow \tau_{i}(t), f_{i j}\left(t+t_{\gamma}\right) \rightarrow f_{i j}(t)$ $(i, l=1,2, \ldots, n ; j, k=1,2, \ldots, m)$ as $\gamma \rightarrow \infty$ uniformly on $R$. Obviously, $\lim _{\gamma \rightarrow+\infty} x_{i}\left(t+t_{\gamma}\right)=$ $g_{i 1}(t), \lim _{\gamma \rightarrow+\infty} y_{j}\left(t+t_{\gamma}\right)=g_{j 2}(t)$. So we have

$$
\begin{aligned}
g_{i 1}^{\prime}(t)= & \lim _{\gamma \rightarrow+\infty} x_{i}^{\prime}\left(t+t_{\gamma}\right) \\
= & \lim _{\gamma \rightarrow+\infty} x_{i}\left(t+t_{\gamma}\right)\left[r_{i}\left(t+t_{\gamma}\right)-\sum_{k=1}^{n} b_{i k}\left(t+t_{\gamma}\right) x_{k}\left(\left(t+t_{\gamma}\right)-\tau_{k}\left(t+t_{\gamma}\right)\right)\right. \\
& \left.-\sum_{k=1}^{m} \frac{c_{i k}\left(t+t_{\gamma}\right) x_{i}\left(t+t_{\gamma}\right) y_{k}^{\alpha}\left(t+t_{\gamma}\right)}{x_{i}^{2}\left(t+t_{\gamma}\right)+f_{i k}\left(t+t_{\gamma}\right)}\right] \\
= & g_{i 1}(t)\left[r_{i}(t)-\sum_{k=1}^{n} b_{i k}(t) g_{k 1}\left(t-\tau_{k}(t)\right)-\sum_{k=1}^{m} \frac{c_{i k}(t) g_{i 1}(t) g_{k 2}^{\alpha}(t)}{g_{i 1}^{2}(t)+f_{i k}(t)}\right], \\
g_{j 2}^{\prime}(t)= & \lim _{\gamma \rightarrow+\infty} y_{j}^{\prime}\left(t+t_{\gamma}\right) \\
= & \lim _{\gamma \rightarrow+\infty} y_{j}\left(t+t_{\gamma}\right)\left[-r_{j}\left(t+t_{\gamma}\right)-\sum_{k=1}^{m} p_{j k}\left(t+t_{\gamma}\right) x_{k}\left(\left(t+t_{\gamma}\right)-\tau_{k}\left(t+t_{\gamma}\right)\right)\right. \\
& \left.+\sum_{k=1}^{n} \frac{q_{i k}\left(t+t_{\gamma}\right) x_{k}^{2}\left(t+t_{\gamma}\right) y_{k}^{\alpha-1}\left(t+t_{\gamma}\right)}{x_{k}^{2}\left(t+t_{\gamma}\right)+f_{k j}\left(t+t_{\gamma}\right)}\right] \\
= & g_{j 2}(t)\left[-r_{j}(t)-\sum_{k=1}^{m} b_{i k}(t) g_{k 2}(t)+\sum_{k=1}^{n} \frac{q_{k j}(t) g_{k 1}^{2}(t) g_{k 2}^{\alpha-1}(t)}{g_{k 1}^{2}(t)+f_{k j}(t)}\right] .
\end{aligned}
$$

From the above, we know $g(t)$ satisfies (1.2), that is, it is a positive almost periodic solution.

Next, we prove that the positive almost periodic solution of system (1.2) is unique.

Let $g(t)>0$ and $\bar{g}(t)>0$ be any two almost periodic solutions of system (1.2), then we claim that $g_{1}(t) \equiv \bar{g}_{1}(t)$ and $g_{2}(t) \equiv \bar{g}_{2}(t)$ for $\forall t \in R$. Otherwise, there is at least one $\xi \in R$ such that $g_{i 1}(\xi) \neq \bar{g}_{i 1}(\xi)$, that is, $\left|g_{i 1}(\xi)-\bar{g}_{i 1}(\xi)\right|:=\delta>0$. Then

$$
\delta=\left|g_{i 1}(\xi)-\bar{g}_{i 1}(\xi)\right|=\lim _{\gamma \rightarrow+\infty}\left|x_{i}\left(\xi+t_{\gamma}\right)-\bar{x}_{i}\left(\xi+t_{\gamma}\right)\right|=\lim _{t \rightarrow+\infty}\left|x_{i}(t)-\bar{x}_{i}(t)\right|>0,
$$

which is a contradiction to (4.18). Thus $\forall t \in R, g_{1}(t) \equiv \bar{g}_{1}(t)$ holds. By the same method, we can prove that $\forall t \in R, g_{2}(t) \equiv \bar{g}_{2}(t)$.

Remark 5.1 If $\tau_{i}(t) \equiv \tau_{i}$, where $\tau_{i}(i=1,2, \ldots, n)$ is a nonnegative constant, then assumptions $\left[H_{2}\right]$ and $\left[H_{3}\right]$ can be redefined. So, we give the following Corollary 5.1. 
Corollary 5.1 Make $\tau_{i}(t) \equiv \tau_{i}$, where $\tau_{i} \geq 0$. If system (1.2) satisfies both $\left[H_{1}\right]$ and the following two conditions:

$$
\begin{aligned}
& \lim _{t \rightarrow+\infty} \inf \left\{-\sum_{k=1}^{m} c_{i k}(t) E_{i k}+\sum_{k=1}^{n} b_{i k}(t)\right. \\
& -\sum_{k=1}^{n}\left[r_{k}(s)+\sum_{j=1}^{n} b_{k j}(s) M_{j}+\sum_{j=1}^{m} \frac{q_{k j}(s) M_{k} N_{j}}{f_{k j}^{l}+m_{k}^{2}}\right] \int_{t}^{t+\tau_{i}} b_{i k}(u) d u \\
& \quad-\sum_{k=1}^{n} \sum_{j=1}^{m} c_{i k}(t) M_{k} E_{k j} \int_{t}^{t+\tau_{i}} b_{i k}(u) d u-\sum_{k=1}^{n} \sum_{j=1}^{n} M_{k} p_{k j}\left(t+\tau_{i}\right) \int_{t+\tau_{i}}^{t+\tau_{k}+\tau_{i}} b_{i k}(u) d u \\
& \left.-\sum_{k=1}^{m} q_{k j}(t) \frac{2 M_{i} N_{j}^{\alpha-1}}{f_{i k}(t)+m_{i}^{2}}\right\}>0, \\
& \lim _{t \rightarrow+\infty} \inf \left\{-\sum_{k=1}^{m} c_{i k}(t) F_{i k}-\sum_{k=1}^{n} \sum_{j=1}^{m} q_{k j}(t) M_{k} E_{k j} \int_{t}^{t+\tau_{i}} b_{i k}(u) d u+\sum_{k=1}^{m} q_{j k}(t)\right\}>0,
\end{aligned}
$$

then system (1.2) has a unique positive almost periodic solution which is globally attractive.

\section{Model simulation}

We give examples to verify the correctness of our theoretical results in this part.

Example 6.1 Consider the following system:

$$
\left\{\begin{aligned}
x^{\prime}(t)= & x(t)[(3+0.1 \sin \sqrt{7} t)-(2-0.1 \sin t) x(t-0.01) \\
& \left.-\frac{(0.05-0.01 \sin t) x(t)}{x^{2}(t)+1} y^{0.2}(t)\right], \\
y^{\prime}(t)= & y(t)[-(0.02+0.01 \sin \sqrt{3} t)-(0.05-0.01 \sin t) y(t) \\
& \left.+\frac{(0.2+0.01 \sin t) x^{2}(t)}{x^{2}(t)+1} y^{-0.8}(t)\right],
\end{aligned}\right.
$$

with the initial conditions $(\phi(0), \psi(0))=(1,1)$ and $(\phi(0), \psi(0))=(2.5,2)$.

By calculation, the parameters of (6.1) meet the conditions of Theorem 3.1 and Corollary 5.1. Using MATLAB, by simulation, time series diagrams of (6.1) are shown in Figure 1. Figure 1 indicates that (6.1) is persistent and has a unique positive almost periodic solution which is globally attractive.

In order to demonstrate the dynamical behaviors of a multispecies predator-prey system, we give the time series diagrams with only three species in system (1.2).

Example 6.2 Consider the following system:

$$
\left\{\begin{aligned}
x_{1}^{\prime}(t)= & x_{1}(t)\left[(1+0.1 \sin \sqrt{7} t)-(1.3-1.1 \sin t) x_{1}(t-0.01)\right. \\
& \left.-(1.4-1.2 \sin t) x_{2}(t-0.01)-\frac{(0.05-0.01 \sin t) x_{1}(t)}{x_{1}^{2}(t)+30} y^{0.5}(t)\right], \\
x_{2}^{\prime}(t)= & x_{2}(t)\left[(1+0.1 \sin \sqrt{7} t)-(1.3-1.1 \sin t) x_{1}(t-0.01)\right. \\
& \left.-(1.4-1.2 \sin t) x_{2}(t-0.01)-\frac{(0.05-0.01 \sin t) x_{2}(t)}{x_{2}^{2}(t)+30} y^{0.5}(t)\right], \\
y^{\prime}(t)= & y(t)[-(0.02+0.01 \sin \sqrt{3} t)-(0.05-0.01 \sin t) y(t) \\
& \left.+\frac{(0.5+0.01 \sin t) x_{1}(t) y^{-0.5}(t)}{x_{1}^{2}(t)+30}+\frac{(0.5+0.01 \sin t) x_{2}(t) y^{-0.5}(t)}{x_{2}^{2}(t)+30}\right],
\end{aligned}\right.
$$




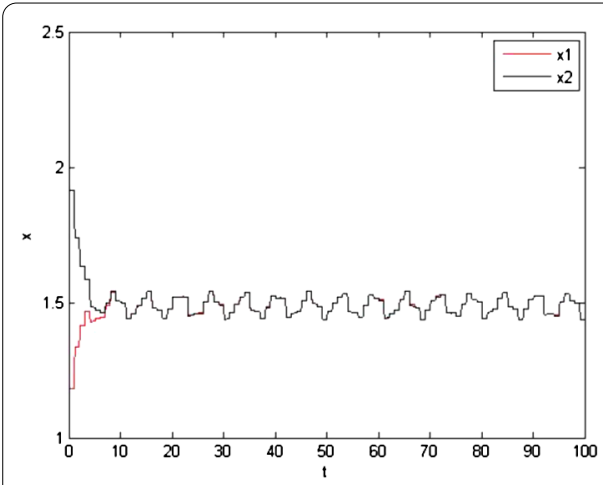

(a)

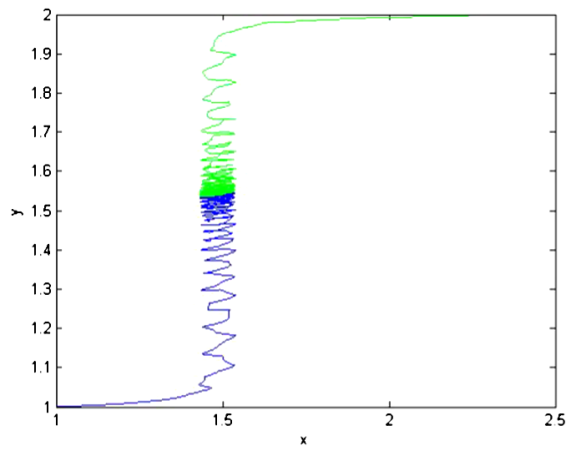

(c)

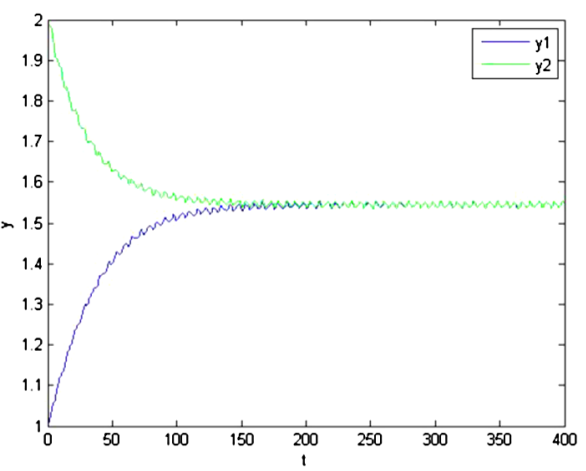

(b)

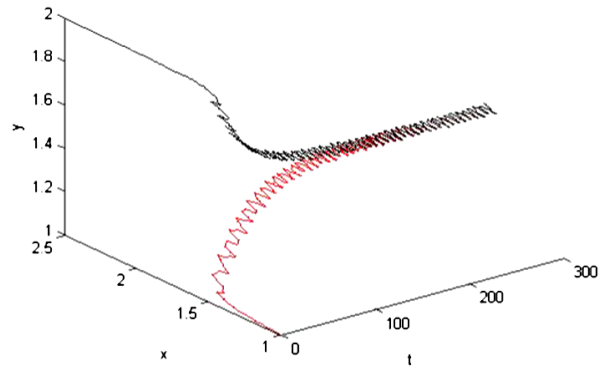

(d)

Figure 1 (a), (b) The time series diagrams with two initial values of prey and predator, respectively. (c) Two-dimensional periodic diagram of predator-prey. (d) Three-dimensional periodic diagram of predator-prey-time.

with the initial conditions $\left(\phi_{1}(0), \phi_{2}(0), \psi(0)\right)=(2,2,2)$ and $\left(\phi_{1}(0), \phi_{2}(0), \psi(0)\right)=(0.1$, $0.1,0.1)$.

By calculation, these parameters of (6.2) meet the conditions of Theorem 3.1 and Corollary 5.1. Using MATLAB, by simulation, time series diagrams of (6.2) are shown in Figure 2. Figure 2 shows that (6.2) is persistent and has a unique positive almost periodic solution which is globally attractive.

\section{Conclusion}

We construct a multispecies predator-prey model with mutual interference and time delays in this article. We obtain the conditions of permanence, global attractivity and uniqueness of positive almost periodic solutions of the system by using the Ascoli theorem, Lebesgue dominated convergence theorem, Lyapunov functions and comparison theorem. Finally, simulation results indicate the correctness of the theoretical results and demonstrate the complex dynamical behaviors of the system.

Compared with Ref. [10], Du only considered the basic dynamics of two species, which can not be effectively promoted and applied in actual production and life. However, we comprehensively integrate the universal phenomenon of multispecies coexistence in the real ecosystem. By studying the dynamics of multispecies predator-prey system, we can better protect the ecosystem and practice the concept of green development. 


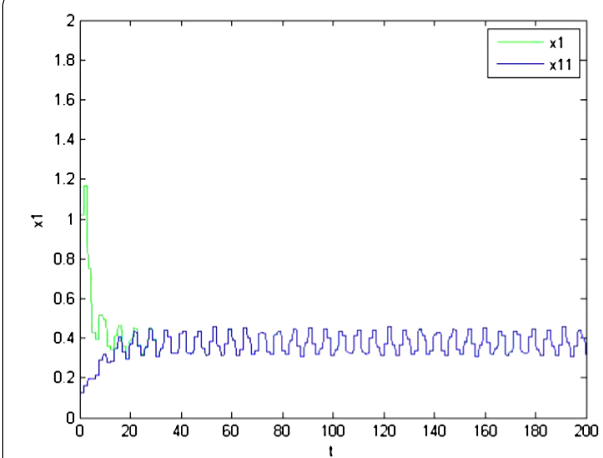

(a)

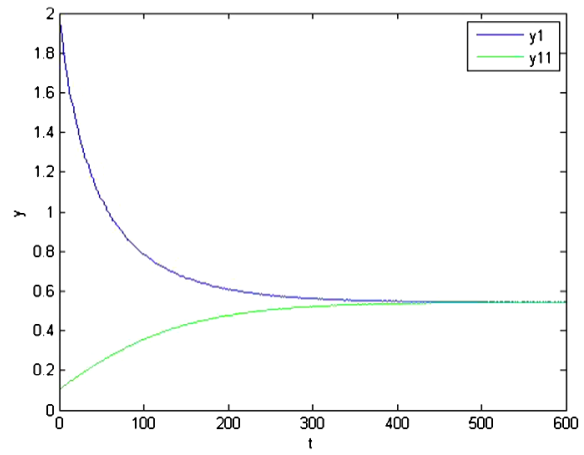

(c)

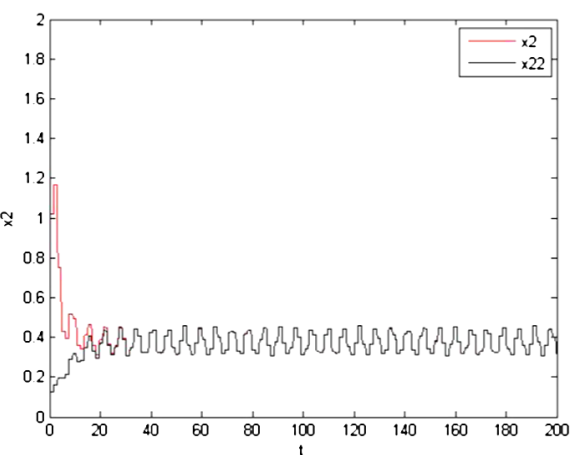

(b)

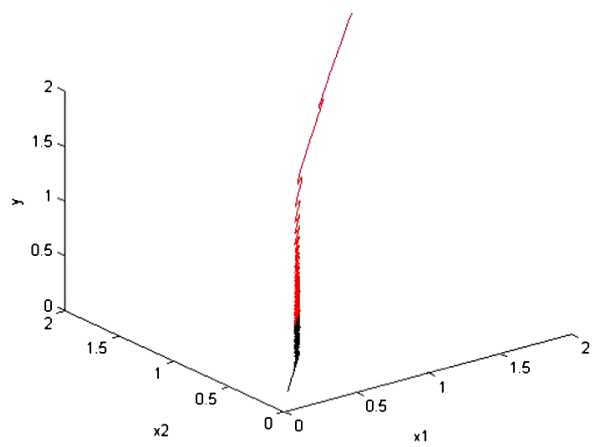

(d)

Figure 2 (a), (b) Time series diagrams with two initial values of prey $x_{i}(i=1,2)$, respectively. (c) The time series diagram with two initial values of predator $y$. (d) Three-dimensional periodic diagram.

\section{Acknowledgements}

This paper is supported by the Natural Science Foundation of Guangxi (2016GXNSFAA380194), National Natural Science Foundation of China (11161015).

\section{Competing interests}

The authors declare that they have no competing interests.

\section{Authors' contributions}

All authors contributed equally in this article. They read and approved the final manuscript.

\section{Authors' information}

QL is now studying for the M.S. degree at Guilin University of Technology, Guilin, China. His research interest is the study of dynamical behaviors and simulation analysis of periodic impulsive differential equations. YS received Ph.D. degree in mathematics from Central South University, Changsha, China. He is a professor of School of Science, Guilin University of Technology, Guilin, China. His research interests are differential equations and dynamical systems. He is mainly engaged in qualitative studies of complex dynamical systems. SZ, ZW and HC are now studying for the M.S. degree at Guilin University of Technology, Guilin, China. Their research interests are stability analysis and numerical simulation of impulsive systems.

\section{Publisher's Note}

Springer Nature remains neutral with regard to jurisdictional claims in published maps and institutional affiliations.

\section{Received: 5 July 2017 Accepted: 8 December 2017 Published online: 21 December 2017}

\section{References}

1. Holling, CS: The functional response of predator to prey density and its role in mimicry and population regulation. Mem. Entomol. Soc. Can. 97(S45), 5-60 (1965)

2. Pei, Y, Chen, L, Zhang, Q, Li, C: Extinction and permanence of one-prey multi-predators of Holling type II function response system with impulsive biological control. J. Theor. Biol. 235(4), 495-503 (2005)

3. Hassel, MP: Density dependence in single-species population. J. Anim. Ecol. 44(1), 283-295 (1975)

4. Guo, $\mathrm{H}$, Chen, X: Existence and global attractivity of positive periodic solution for a Volterra model with mutual interference and Beddington-DeAngelis functional response. Appl. Math. Comput. 217, 5830-5837 (2011) 
5. Lin, X, Chen, F: Almost periodic solution for a Volterra model with mutual interference and Beddington-DeAngelis functional response. Appl. Math. Comput. 214(2), 548-556 (2009)

6. Wang, K: Permanence and global asymptotical stability of a predator-prey model with mutual interference. Nonlinear Anal., Real World Appl. 12(2), 1062-1071 (2011)

7. Lv, Y, Du, Z: Existence and global attractivity of a positive periodic solution to a Lotka-Volterra model with mutual interference and Holling III type functional response. Nonlinear Anal., Real World Appl. 12(6), 3654-3664 (2011)

8. Zeng, G, Wang, F, Nieto, JJ: Complexity of a delayed predator-prey model with impulsive harvest and Holling type II functional response. Adv. Complex Syst. 11(1), 77-97 (2008)

9. Wang, K, Zhu, Y: Permanence and global asymptotic stability of a delayed predator-prey model with Hassell-Varley type functional response. Bull. Iran. Math. Soc. 37(3), 197-215 (2011)

10. Du, Z, Lv, Y: Permanence and almost periodic solution of a Lotka-Volterra model with mutual interference and time delays. Appl. Math. Model. 37(3), 1054-1068 (2013)

11. Shao, Y, Dai, B, Luo, Z: The dynamics of an impulsive one-prey multi-predators system with delay and Holling-type II functional response. Appl. Math. Comput. 217(6), 2414-2424 (2010)

12. Bahaa, GM: Fractional optimal control problem for differential system with delay argument. Adv. Differ. Equ. (2017). https://doi.org/10.1186/s13662-017-1121-6

13. Wang, B, Cheng, J, Al-Barakati, A, Fardoun, HM: A mismatched membership function approach to sampled-data stabilization for T-S fuzzy systems with time-varying delayed signals. Signal Process. 140, 161-170 (2017)

14. Cheng, J, Park, JH, Zhang, L, Zhu, Y: An asynchronous operation approach to event-triggered control for fuzzy Markovian jump systems with general switching policies. IEEE Trans. Fuzzy Syst. (2016). https://doi.org/10.1109/TFUZZ.2016.2633325

15. Meng, X, Zhao, S, Zhang, W: Adaptive dynamics analysis of a predator-prey model with selective disturbance. Appl. Math. Comput. 266, 946-958 (2015)

16. Aghajanzadeh, O, Sharif, M, Tashakori, S, Zohoor, H: Nonlinear adaptive control method for treatment of uncertain hepatitis B virus infection. Biomed. Signal Process. Control 38, 174-181 (2017)

17. Rui, J, Liu, B: Almost-periodic solutions of an almost-periodically forced wave equation. J. Math. Anal. Appl. 451(2) 629-658 (2017)

18. Meng, X, Chen, L: Almost periodic solution of non-autonomous Lotka-Volterra predator-prey dispersal system with delays. J. Theor. Biol. 243(4), 562-574 (2006)

19. Chen, X: Almost periodic solutions of nonlinear delay population equation with feedback control. Nonlinear Anal., Real World Appl. 8(1), 62-72 (2007)

20. Zhou, Z: Global attractivity and periodic solution of a discrete multispecies cooperation and competition predator-prey system. Discrete Dyn. Nat. Soc. 2011, Article ID 835321 (2011)

21. Qiao, Y, Zhou, Z: Existence of positive solutions of singular fractional differential equations with infinite-point boundary conditions. Adv. Differ. Equ. (2017). https://doi.org/10.1186/s13662-016-1042-9

22. Miao, Z, Chen, F, Liu, J, Pu, L: Dynamic behaviors of a discrete Lotka-Volterra competitive system with the effect of toxic substances and feedback controls. Adv. Differ. Equ. (2017). https://doi.org/10.1186/s13662-017-1130-5

23. Chen, F: Almost periodic solution of the non-autonomous two-species competitive model with stage structure. Appl. Math. Comput. 181(1), 685-693 (2006)

24. Fink, AM: Almost Periodic Differential Equations. Springer, Berlin (1974)

25. Qi, W, Dai, B: Almost periodic solution for $n$-species Lotka-Volterra competitive system with delay and feedback controls. Appl. Math. Comput. 200(1), 133-146 (2008)

26. Gopalsamy, K: Stability and Oscillations in Delay Differential Equations of Population Dynamics. Springer, Berlin (1992)

27. Zeng, G, Chen, L, Sun, L, Liu, Y: Permanence and the existence of the periodic solution of the non-autonomous two-species competitive model with stage structure. Adv. Complex Syst. (2004). https://doi.org/10.1142/S0219525904000238

28. Shen, C: Permanence and global attractivity of the food-chain system with Holling IV type functional response. Appl. Math. Comput. 194(1), 179-185 (2007)

29. Zhu, Y: Uniformly persistence of a predator-prey model with Holling III type functional response. Int. J. Comput. Math. Sci. 4(7), 355-357 (2010)

\section{Submit your manuscript to a SpringerOpen ${ }^{\circ}$ journal and benefit from:}

- Convenient online submission

- Rigorous peer review

- Open access: articles freely available online

- High visibility within the field

- Retaining the copyright to your article

Submit your next manuscript at $\gg$ springeropen.com 